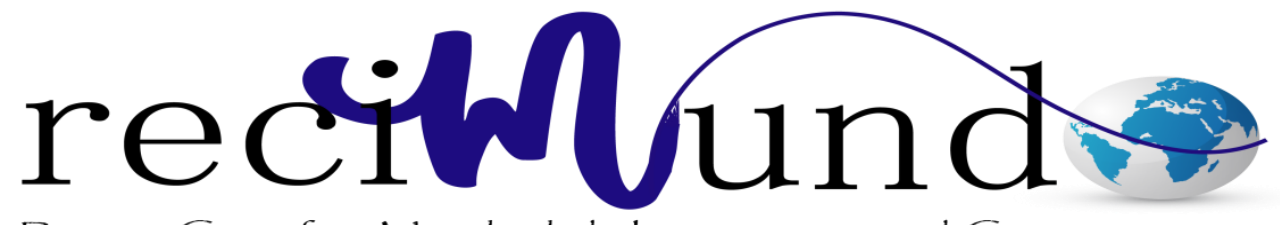

Revista Científica Mundo de la Investigación y el Conocimiento

Diego Andrés Pazmiño Romero ${ }^{a}$; Georgina Andalira Jácome Lara ${ }^{b}$

Oferta turística de la parroquia Balsapamba y su efecto en la percepción de la demanda

Revista Científica Mundo de la Investigación y el Conocimiento. Vol. 1 núm., 4, septiembre, 2017, pp. 919-933

DOI: 10.26820/recimundo/1.4.2017.919-933

Editorial Saberes del Conocimiento

a. Universidad Técnica de Babahoyo; dpazmino@utb.edu.ec

b. Universidad Técnica de Babahoyo; gjacome@utb.edu.ec 


\section{Oferta turística de la parroquia Balsapamba y su efecto en la percepción de la demanda}

Vol. 1, núm. 4., (2017)

Diego Andrés Pazmiño Romero; Georgina Andalira Jácome Lara

\section{RESUMEN}

El propósito de esta investigación es identificar la incidencia que tienen las actuales acciones realizadas por la empresa privada y pública para mejorar la oferta turística de la parroquia Balsapamba. Para posteriormente evaluar de manera global la percepción del destino turístico Balsapamba como marca.

En este estudio se hallan factores favorables que permitirían el desarrollo de este sector, por su ubicación cercana al mercado potencial Guayaquil, además de un clima privilegiado y singular. Lo que puede ser utilizado como una fortaleza al realizarse una estrategia de mercado para posicionar el destino.

El proyecto en su ejecución es un generador de oportunidades para la micro, pequeña y mediana empresa que operan dentro de la cadena de valor del turismo, tanto para quienes deseen emprender con nuevos proyectos como para los micro empresarios que buscan diversificar e innovar sus servicios.

Los sectores beneficiados de la ejecución de este proyecto son especialmente las comunidades rurales del cantón San Miguel del Bolívar y sectores aledaños, que participen directa o indirectamente de la industria del turismo, de forma dependiente o independiente mediante el emprendimiento.

Este estudio será un ensayo que podría servir de ejemplo a muchas comunidades que tienen similares fortalezas que les permitirá ver en el turismo una oportunidad de desarrollo sustentable.

Palabras clave: Oferta; turismo; percepción; demanda. 


\title{
Oferta turística de la parroquia Balsapamba y su efecto en la percepción de la demanda
}

Vol. 1, núm. 4., (2017)

Diego Andrés Pazmiño Romero; Georgina Andalira Jácome Lara

\begin{abstract}
The purpose of this research is to identify the impact of the current actions carried out by the private and public companies to improve the tourist offer of the Balsapamba parish. In order to evaluate in a global way the perception of the Balsapamba tourist destination as a brand.

In this study we find favorable factors that would allow the development of this sector, because of its location near the potential market Guayaquil, in addition to a privileged and unique climate. What can be used as strength when performing a market strategy to position the destination.

The project in its execution is a generator of opportunities for micro, small and medium enterprises operating within the value chain of tourism, both for those who wish to undertake new projects and for micro entrepreneurs seeking to diversify and innovate their services.

The sectors benefiting from the implementation of this project are especially the rural communities of the canton San Miguel del Bolívar and surrounding sectors, which participate directly or indirectly in the tourism industry, in a dependent or independent way through entrepreneurship.

This study will be a test that could serve as an example to many communities that have similar strengths that will allow them to see in tourism an opportunity for sustainable development.
\end{abstract}

Keywords: Offer; tourism; perception; demand. 


\section{Oferta turística de la parroquia Balsapamba y su efecto en la percepción de la demanda}

Vol. 1, núm. 4., (2017)

Diego Andrés Pazmiño Romero; Georgina Andalira Jácome Lara

\section{Introducción.}

Desde hace más de diez años el Ecuador ha tomado algunas iniciativas claras y significativas inversiones que fortalezcan la competitividad de la industria del turismo nacional como la realización del evento Miss Universo 2004, La campaña mundial All You Needs Ecuador y la cuña publicitaria en el Súper Tazón 2015. Hitos de la historia y desarrollo de esta industria en el país, con el objetivo de llevar a Ecuador como potencia turística a lo más alto de las ofertas en el mercado mundial, y evidencia de esto es que el Ecuador fue reconocido con catorce premios de los que ocho corresponden a la categoría Sudamérica y seis a Ecuador.

"El turismo es el segundo generador de empleo en el Ecuador" Sandra Naranjo, ministra de Turismo del Ecuador (Diario El Comercio, 18 de Noviembre 2014)

Bolívar una pequeña provincia de la sierra ecuatoriana con ciudades de arquitectura colonial como Guaranda y San Miguel donde sus habitantes llevan una vida conservadora y alejada de la bulla y el estrés de las grandes ciudades, sus pobladores que hasta hace pocos años veían sus comunidades olvidadas y de le tardadas economías han visto con asombro como se han convertido estas localidades por su ubicación geográfica en un sitio ideal para realizar actividades turísticas que son del interés de los visitantes y transeúntes de la provincia los que llevan con aprecio un recuerdo de su visita al conocer sus paisajes, y centros de ocio haciendo estos recorridos desde en paseos de "chiva" o simplemente caminar y explorar cada una de sus cinco cascadas. Sus atractivos no pasan desapercibidos a los ojos de los turistas, pues es el paso obligado por estar asentado cerca de la carretera principal. En el recinto El Cristal, ubicado a pocos metros de la vía hacia Guaranda, está el Parque Acuático, un sitio con perfil apropiado, 


\section{Oferta turística de la parroquia Balsapamba y su efecto en la percepción de la demanda}

Vol. 1, núm. 4., (2017)

Diego Andrés Pazmiño Romero; Georgina Andalira Jácome Lara

rodeado de paisajes, ríos y diversidad. En los últimos meses el turismo comenzó a reactivarse, ante el asombro de sus habitantes que ven desfilar caravanas de carros interprovinciales y particulares. También se conoce por tradición a la parroquia Salinas, el centro turístico Las Cochas, el santuario del Guaico y las cascadas que están conectadas a la vía.

Este proyecto de análisis pretende descubrir el impacto económico que puede tener la industria del turismo como espectro generador de desarrollo y empleo en la economía de una pequeña localidad de la provincia del Bolívar, la Parroquia Balsapamba del Cantón San Miguel. Considerando la oferta turística su clasificación y repercusión en el entorno de su comuna con empleos directos e indirectos y la creación de pequeñas y medianas empresas que ofrezcan servicios complementarios o principales de la cadena de valor en la industria de servicios turísticos.

\section{Desarrollo}

La Parroquia Balsapamba perteneciente al Cantón San Miguel del Bolívar es una pequeña comuna de características limitadas en sus servicios, como la falta de agua potable, internet, energía eléctrica (regular), centros de salud, y centros de educación con un servicio digno, carreteras que en buen estado y sean favorables al comercio y que permitan el acceso comercial a la localidad, estos y otros son algunos indicadores sociales o de calidad de vida que caracterizan la pobreza de sus habitantes.

La lenta y desapercibida economía que agonizaba en la parroquia era basada en actividades agrícolas y pecuarias, como el cultivo de naranja, la crianza de aves y porcinos para 


\section{Oferta turística de la parroquia Balsapamba y su efecto en la percepción de la demanda}

Vol. 1, núm. 4., (2017)

Diego Andrés Pazmiño Romero; Georgina Andalira Jácome Lara

la producción de su carne. Sin embargo la cadena de valor implícita en este tipo de operaciones no generaba mayor empleo, ni ingresos que los permitidos por la variabilidad de precios del mercado, y sus volúmenes no son suficientes para la demanda local.

El mejoramiento del estado de las carreteras que unen a Balsapamba con Guaranda y Babahoyo han permitido que los pobladores de localidades cercanas como Guayaquil, Duran, Milagro, Babahoyo, Guaranda, San Miguel, Vinces y otros. Visiten y disfruten de sus ofertas turísticas naturales tales como cascadas, ríos, avistamiento de orquídeas y paisajes del subtropical andino. Creando el interés en los inversionistas públicos y privados en mejorar la oferta creando balnearios artificiales, comedores, hosterías, y locales de artesanías o actividades diversas y relacionados con el turismo.

Situación que ha permitido que muchos pobladores pasen a formar parte de la masa laboral activa sin perjudicar su entorno y respetando sus tradiciones, aprovechando los beneficios que la naturaleza generosamente ha puesto en esta localidad de la Provincia del Bolívar.

Una administración responsable de las empresas que forman parte de las ofertas turísticas que tiene la parroquia permitirá el continuo desarrollo de esta localidad. Esto siempre que se respete su naturaleza o el uso de ella sea amistoso con el medio ambiente, caso contrario será un fuente de riqueza no renovable que se ira deteriorando con el uso irresponsable de parte de usuarios y administradores de los negocios protagonistas de esta cadena de valor.

Este proyecto pretende identificar la importancia que ha tenido la actividad turística para la parroquia con el propósito de concientizar a todos los actores de su entorno y motivar al uso y 


\section{Oferta turística de la parroquia Balsapamba y su efecto en la percepción de la demanda}

Vol. 1, núm. 4., (2017)

Diego Andrés Pazmiño Romero; Georgina Andalira Jácome Lara

administración de su naturaleza de manera técnica y responsable para el beneficio de sus pobladores. Para lo que se considera necesario contestar:

¿Qué gestiones de las empresas públicas y privadas inciden en el desarrollo de la oferta turística de la parroquia balsapamba?

¿Por qué la empresa privada no innova en servicios de alojamiento en la parroquia Balsapamba?

Son los principales motivos de investigación del estudio, para lo cual se analizaron de forma estadística mediante el resultado de encuestas realizadas a quienes protagonizan las actividades turísticas en el sector privado. Para determinar las gestiones de las empresas que inciden en el desarrollo de la oferta turística de la parroquia Balsapamba. Con este análisis se pretende:

Establecer las circunstancias necesarias para que la empresa privada innove en servicios de alojamiento en la parroquia Balsapamba

Establecidas las circunstancias requeridas para la innovación de servicios en los negocios dedicados al alojamiento y derivados del turismo, se diversificará la oferta a través de actividades de recreación relacionadas al turismo.

En esta investigación estudiaremos situaciones vigentes, sin que estas sean motivadas a llegar a algún resultado de manera tendenciosa, se investigará el acontecimiento tal como sucede, 


\section{Oferta turística de la parroquia Balsapamba y su efecto en la percepción de la demanda}

Vol. 1, núm. 4., (2017)

Diego Andrés Pazmiño Romero; Georgina Andalira Jácome Lara

para su delineación específica y las reacciones de cada variable en su interacción. Con lo que se pretende encontrar una alternativa que permita generar soluciones al foco de estudio.

El estudio que se realiza procura describir las escenas del fenómeno tal y como acontecen siendo estas interpretadas mediante información compuesta por datos cuantitativos que darán la posibilidad de analizar el comportamiento de la demanda en función a la oferta. Y es en base a las cifras que representan las interacciones de cada una de las variables en el estudio que nos brindará la posibilidad de tener resultados muy cercanos a los sucesos investigados.

En base a lo manifestado podemos determinar que esta investigación será de tipo descriptiva y no experimental puesto que son sucesos no incitados si no de carácter espontaneo.

\section{Resultados}

1.- ¿Cómo califica su visita a Balsapamba?

- Percepción de calidad de servicios turísticos en Balsapamba.

\begin{tabular}{|l|c|c|}
\hline \multicolumn{1}{|c|}{ OPINIÓN } & ENCUESTADOS & PORCENTAJE \\
\hline Muy Bueno & 8 & $57.14 \%$ \\
\hline Bueno & 3 & $21.43 \%$ \\
\hline Regular & 1 & $7.14 \%$ \\
\hline Malo & 1 & $7.14 \%$ \\
\hline Muy Malo & 1 & $7.14 \%$ \\
\hline TOTAL & $\mathbf{1 4}$ & $\mathbf{1 0 0 . 0 0 \%}$ \\
\hline
\end{tabular}

Fuente: Encuesta 
Oferta turística de la parroquia Balsapamba y su efecto en la percepción de la demanda

Vol. 1, núm. 4., (2017)

Diego Andrés Pazmiño Romero; Georgina Andalira Jácome Lara

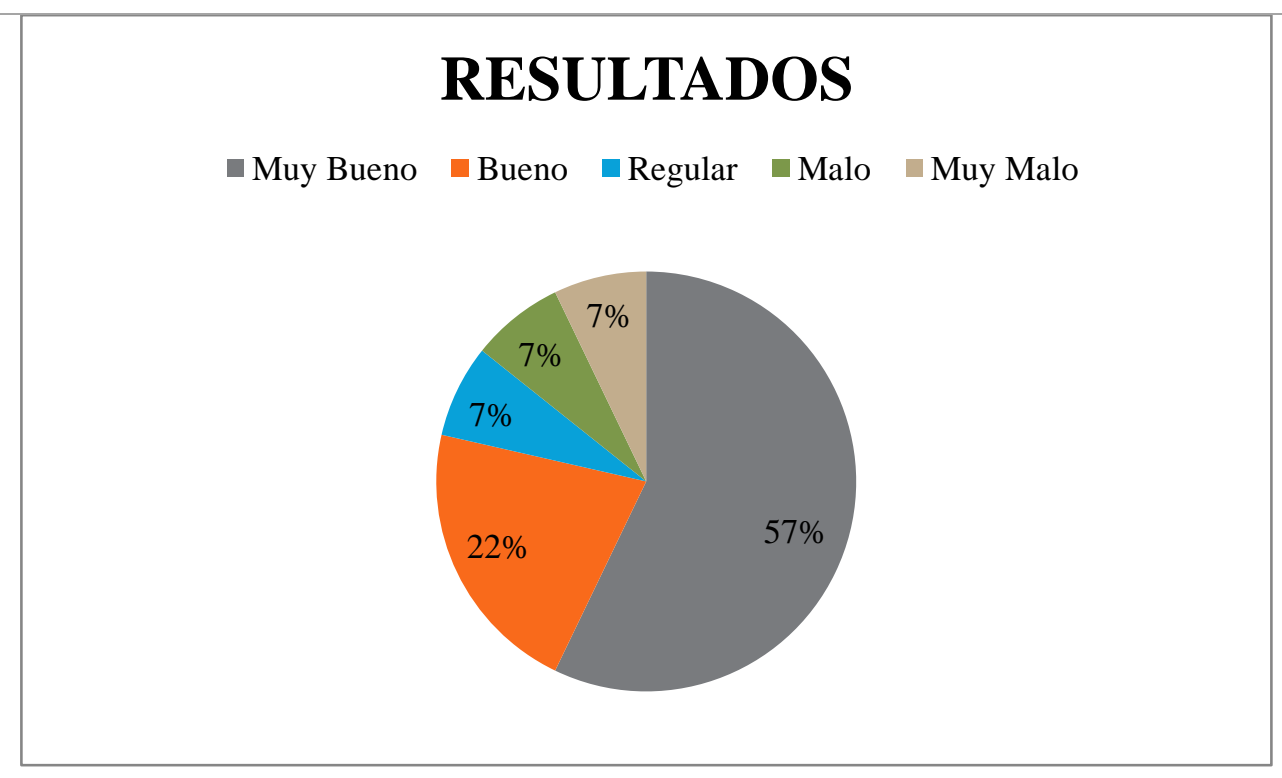

Revela que la percepción del cliente sobre Balsapamba como destino turístico es elevada, ya que cerca del $80 \%$ de los visitantes ha calificado con bueno o muy bueno su experiencia en el sector estudiado. Esto demuestra que si un mayor porcentaje del mercado potencial visitara Balsapamba lo tendría en su "top of the mind" de destinos turísticos locales.

2.- ¿Qué Lugares visitó?

Atracciones turísticas visitadas por el mercado actual

\begin{tabular}{|l|c|c|}
\hline \multicolumn{1}{|c|}{ OPINIÓN } & ENCUESTADOS & PORCENTAJE \\
\hline Parque Acuático & 61 & $92.42 \%$ \\
\hline Castillo Suizo & 42 & $63.64 \%$ \\
\hline Cascada Milagrosa & 64 & $96.97 \%$ \\
\hline Cascada de Angas & 51 & $77.27 \%$ \\
\hline Cascada Mariposa Azul & 32 & $48.48 \%$ \\
\hline Paseo en Chiva & 24 & $36.36 \%$ \\
\hline Canopy & 14 & $21.21 \%$ \\
\hline Trapiche & 12 & $18.18 \%$ \\
\hline Otro & 11 & $16.67 \%$ \\
\hline Ninguno & 2 & $3.03 \%$ \\
\hline
\end{tabular}


Oferta turística de la parroquia Balsapamba y su efecto en la percepción de la demanda

Vol. 1, núm. 4., (2017)

Diego Andrés Pazmiño Romero; Georgina Andalira Jácome Lara

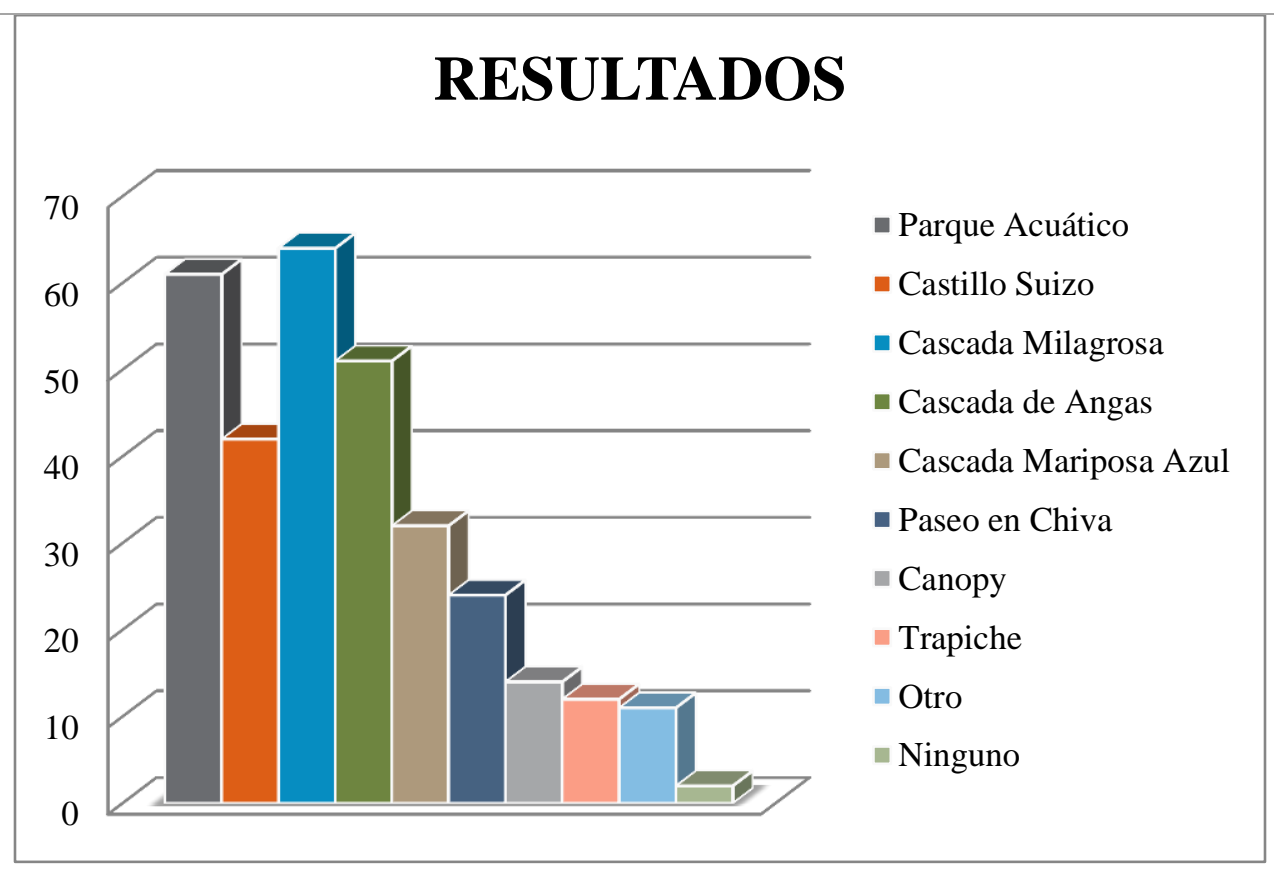

(Tomando en cuenta que se puede marcar más de una respuesta) las atracciones de mayor aceptación son las del Parque Acuático y La Cascada Milagrosa, los usuarios suelen pasar la mayor parte del día en el lugar debido a que cuentan con música, comida, cerveza y lugar para bañarse a precios módicos. 
Oferta turística de la parroquia Balsapamba y su efecto en la percepción de la demanda

Vol. 1, núm. 4., (2017)

Diego Andrés Pazmiño Romero; Georgina Andalira Jácome Lara

\section{3.- ¿Qué actividades le gustaría que hacer en Balsapamba en su próxima visita?}

Expectativas de Opciones de actividades turísticas en Balsapamba.

\begin{tabular}{|l|c|c|}
\hline \multicolumn{1}{|c|}{ OPINIÓN } & ENCUESTADOS & PORCENTAJE \\
\hline Museos & 1 & $1.52 \%$ \\
\hline Balnearios & 64 & $96.97 \%$ \\
\hline Cascadas & 61 & $92.42 \%$ \\
\hline Hiking & 23 & $34.85 \%$ \\
\hline Canopy & 21 & $31.82 \%$ \\
\hline Caballos & 14 & $21.21 \%$ \\
\hline M. Bike & 6 & $9.09 \%$ \\
\hline Trapiches & 4 & $6.06 \%$ \\
\hline Pescar & 5 & $7.58 \%$ \\
\hline Bailar & 62 & $93.94 \%$ \\
\hline Otra & 23 & $34.85 \%$ \\
\hline Ninguna & 4 & $6.06 \%$ \\
\hline
\end{tabular}


Oferta turística de la parroquia Balsapamba y su efecto en la percepción de la demanda

Vol. 1, núm. 4., (2017)

Diego Andrés Pazmiño Romero; Georgina Andalira Jácome Lara

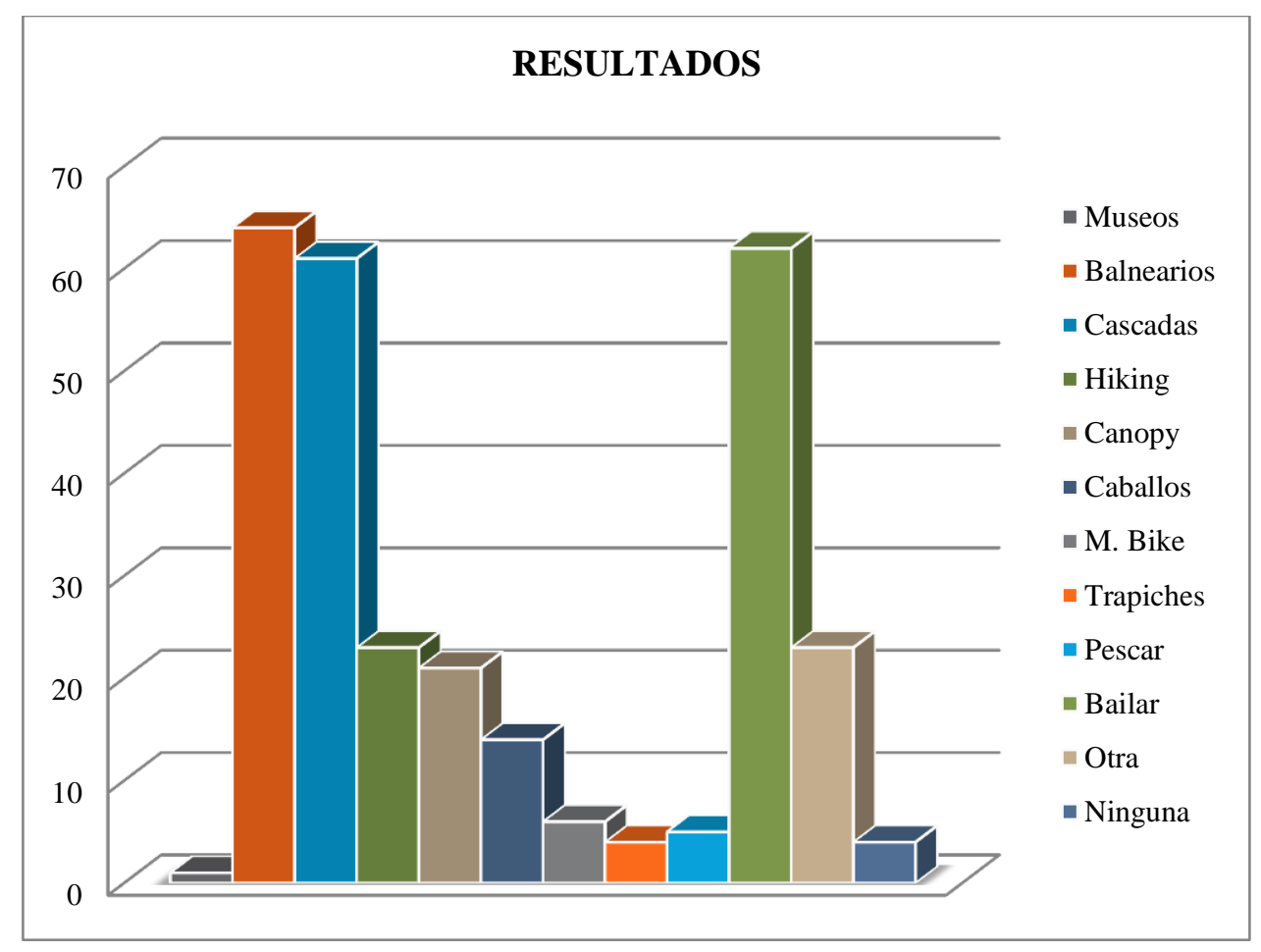

Observando el Cuadro queda claro que las expectativas del mercado actual difieren mucho de las del mercado meta. Los clientes que conforman el mercado actual muestran altos intereses por los Balnearios y los lugares para Bailar ya que esa es la clase de diversión que buscan y las cascadas tienen gran acogida puesto que el acceso a las mismas es gratis. Las demás actividades, en el mejor de los casos alcanzan niveles cercanos al 35\% y las demás no atraen casi ningún interés por parte de los turistas actuales. 


\section{Oferta turística de la parroquia Balsapamba y su efecto en la percepción de la demanda}

Vol. 1, núm. 4., (2017)

Diego Andrés Pazmiño Romero; Georgina Andalira Jácome Lara

\section{Análisis:}

Después de las entrevistas con miembros de la Oferta Turística se establece que el mercado actual no tiene las características adecuadas para que se suscite una evolución significativa del sector. Se determina que los clientes actuales son de un nivel socioeconómico medio bajo proveniente de Guayaquil y Babahoyo con niveles de consumo diarios de entre veinte y cinco y treinta y cinco dólares, que están por debajo de los niveles necesarios para un crecimiento atractivo del sector. Las estrategias comunicacionales de la Oferta se basa netamente en el "boca oído boca", que si bien es cierto que la efectividad de este método es alta por la fidelidad de la información percibida por el receptor no es eficiente porque su propagación es pobre y se limita al nivel socioeconómico del mercado actual. Este método es utilizado por el desconocimiento de otras herramientas y el bajo presupuesto para esta actividad.

Actualmente el Sector Público realiza actividades directas e indirectas para mejorar la Oferta Turística de Balsapamba. Como Indirectas se cataloga a las obras públicas en vialidad realizadas por los GAD de Bolívar y Los Ríos que anuqué no fueron planificadas con este fin ayudan a que los clientes logren trasladarse fácilmente y esto promueve la afluencia de visitantes. Como actividades directas se califican a las acciones ejecutadas por la Junta Parroquial que se limitan a promocionar el carnaval por medio de la elección de la Reina del Carnaval y la organización de las Comparsas. En este grupo también se incluye al mantenimiento del Parque Acuático cuya administración está a cargo del GAD de Bolívar. Estas acciones son percibidas como insuficientes por los miembros de la Oferta Turística ya que a excepción del 


\section{Oferta turística de la parroquia Balsapamba y su efecto en la percepción de la demanda}

Vol. 1, núm. 4., (2017)

Diego Andrés Pazmiño Romero; Georgina Andalira Jácome Lara

carnaval no se hace una correcta promoción de Balsapamba como destino turístico y esta responsabilidad recae únicamente en un sector privado sin la solvencia necesaria para invertir.

Las acciones tomadas por el Sector Privado son escasas, como se expresó anteriormente, la difusión del destino turístico es sumamente básica y poco eficiente, mientras que la estrategia comercial se basa en ofrecer precios bajos pero con los volúmenes de clientes percibidos esta estrategia se vuelve poco efectiva. No hay una visión clara de que acciones tomar por parte de los empresarios y su visión del negocio es limitada finalmente podemos determinar que si no hay cambios en la difusión del destino turístico se prevé un estancamiento del sector o incluso una contracción.

\section{Conclusiones.}

Los ofertas turísticas de la sierra Ecuatoriana lideran entre las regiones preferidas como destinos turísticos entre los ciudadanos de Guayaquil.

Los turistas internos viajan por placer y buscan nuevas actividades de recreación.

Los turistas que conocen Balsapamba están conformes con el servicio de alojamiento que han recibido, y aunque no conocen acerca de las actividades de recreación disponibles en la parroquia, estarían dispuestos a volver y comentar sus buenas experiencias con los demás.

Los turistas que aún no conocen Balsapamba están interesados en saber más información y visitar el destino turístico. 


\section{Oferta turística de la parroquia Balsapamba y su efecto en la percepción de la demanda}

Vol. 1, núm. 4., (2017)

Diego Andrés Pazmiño Romero; Georgina Andalira Jácome Lara

El nivel de gasto que realiza el actual turista que visita Balsapamba no permite entregar servicios con mayor valor agregado.

Los esfuerzos que han realizado la empresa privada carecen de orientación al mercado y son dispersos.

Las acciones que han realizado los gobiernos seccionales que han servido para el crecimiento del turismo, carecen de sustentabilidad mediante la promoción comunicacional.

\section{Bibliografía.}

Cruz, M. \& Palma, K. (2009).Formación de una microempresa de turismo vacacional, su estructura organizativa, el manual de funciones y directrices generales para la atención a los clientes, ciudad de Babahoyo, provincia de Los Ríos. Tesis de grado previo a la obtención del título de ingeniero comercial, Universidad Técnica de Babahoyo, Babahoyo.

Kotler, P; Bowen, J; Makens, J; Garcia de Madariaga, J; Flores,J. (2011). Marketing Turistico (5 edición). Madrid: Pearson Educacion, S.A.

Stanton, W; Etzel, M; Walker, B.(2007). Fundamentos de Marketing(14 edición).Mexico DF:Mc Graw-Hill Interamericana.

Insulza, J. (2011) Discurso del secretario General de la OEA, en la inauguración del XIX CIT. Informe Memoria Turismo: Un desafío frente a la pobreza XIX Congreso Interamericano de Turismo, Septiembre 2011, 44

Subsecretaria de Información y Comunicación Turística y Ediecuatorial. (2012) Acciones que fortalecen al turismo del país. Revista del Ministerio de Turismo del Ecuador,(12657) 30.

Subsecretaria de Información y Comunicación Turística y Ediecuatorial. (2012) Turismo Consciente. Revista del Ministerio de Turismo del Ecuador,(12453) 30.

Ministerio de Obras Públicas. (2014). Plan Anual de Inversión Publica 2012, http://www.mop.gob.ec 